Source: Ţəlu, Ş., Bramowicz, M., Kulesza, S., Shafiekhani, A., Ghaderi, A., Mashayekhi, F., \& Solaymani, S. (2015). Microstructure and tribological properties of FeNPs@a-C:H films by micromorphology analysis and fractal geometry. Industrial and Engineering Chemistry Research, 54(33), 8212-8218. doi:10.1021/acs.iecr.5b02449

\title{
Microstructure and tribological properties of FeNPs@a-C:H films by micromorphology analysis and fractal geometry
}

\author{
Ştefan Ţălu ${ }^{1}$, Miroslaw Bramowicz ${ }^{2}$, Slawomir Kulesza ${ }^{3}$, Azizollah Shafiekhani ${ }^{4,5}$ \\ Atefeh Ghaderi ${ }^{6}$, Fatemeh Mashayekhi ${ }^{7}$, Shahram Solaymani ${ }^{6}$
}

${ }^{1}$ Faculty of Mechanical Engineering, Department of AET, Discipline of Descriptive Geometry and Engineering Graphics, Technical University of Cluj-Napoca, 103-105 B-dul Muncii Street, 400641 Cluj-Napoca, Romania.

${ }^{2}$ Faculty of Technical Sciences, University of Warmia and Mazury in Olsztyn, Oczapowskiego 11, 10-719 Olsztyn, Poland.

${ }^{3}$ Faculty of Mathematics and Computer Science, University of Warmia and Mazury in Olsztyn, Sloneczna 54, 10710 Olsztyn, Poland.

${ }^{4}$ Department of Physics, Faculty of Physics and Chemistry, Alzahra University, Vanak, Tehran, Iran.

${ }^{5}$ School of Physics, Institute for Research in Fundamental Sciences, P.O. Box 19395-5531, Tehran, Iran.

${ }^{6}$ Young Researchers and Elite Club, Kermanshah Branch, Islamic Azad University, Kermanshah, Iran.

${ }^{7}$ Plasma Physics Research Center, Science and Research Branch, Islamic Azad University, Tehran, Iran.

\section{Abstract}

This paper analyzes the three-dimensional (3D) surface texture of amorphous hydrogenated carbon films with sputtered iron nanoparticles (FeNPs@a-C:H) deposited by a radio-frequency plasma-enhanced chemical vapor deposition method on the quartz substrates. The prepared FeNPs@a-C:H films were used as research materials. The synthesized samples were deposited at four different pressures of 2.5, $3,3.35$, and $3.5 \mathrm{~N} / \mathrm{m} 2$ in an acetylene gas atmosphere. The Fe and $\mathrm{C}$ contents of the thin films were obtained from X-ray photoelectron spectroscopy. The X-ray diffraction profile and electron diffraction pattern indicate that iron nanoparticles with body-centered-cubic crystalline structure are formed in these films. The localized surface plasmon resonance peak that is the signature of the existence of the iron core nanoparticles appears in visible spectra of these films. The sample surface images were recorded using an atomic force microscope operating in a noncontact mode and analyzed to reveal the statistical, fractal, and functional surface properties of prepared samples. Analysis of the 3D surface texture is essential for the correct interpretation of surface topographic features as well as its functional role for the test surface. It also helps to understand the relationship between the surface topography and functional properties. 